Case

Report

\title{
Left Ventricular Outflow Tract Obstruction of Double Valve Re-Replacement Using Bioprosthesis
}

\author{
Yoshimori Araki, MD, PhD, Chikao Teramoto, MD, Yasunobu Konishi, MD, \\ Takafumi Terada, MD, and Osamu Kawaguchi, MD, PhD
}

\begin{abstract}
We present a case of left ventricular outflow tract (LVOT) obstruction after double valve re-replacement with bioprostheses. A 72-year-old man, who had undergone double valve replacement (DVR) with bioprosthetic valves 9 years previously, underwent re-replacement of valves because of structural valve deterioration. However, owing to LVOT obstruction related to the bioprosthesis in the mitral position, acute pulmonary edema occurred immediately after surgery. LVOT obstruction was diagnosed by emergent cardiac catheterization. So prompt re-replacement surgery using a mechanical prosthesis was performed.
\end{abstract}

Keywords: double valve replacement, left ventricular outflow tract obstruction, bioprosthesis

\section{Introduction}

It has been noted that a bioprosthesis implanted in the mitral position can cause left ventricular outflow tract (LVOT) obstruction. ${ }^{1-3)}$ However, the location of the bioprosthesis makes it very difficult to diagnose such obstruction. We report a case of LVOT obstruction diagnosed by emergent cardiac catheterization after double valve replacement (DVR) with bioprostheses.

\section{Case Report}

The patient was a 72-year-old man who had undergone DVR with bioprosthetic valves 9 years previously because of infectious endocarditis, whereby the bioprostheses used were a 27-mm Mosaic (Medtronic, Minneapolis,

Department of Cardiac Surgery, Toyota Kosei Hospital, Toyota, Aichi, Japan

Received: April 10, 2018; Accepted: July 15, 2018

Corresponding author: Yoshimori Araki, MD, PhD. Department of Cardiac Surgery, Toyota Kosei Hospital, 500-1 Ibohara, Josui-Cho, Toyota, Aichi 470-0343, Japan

Email: yaraki@med.nagoya-u.ac.jp

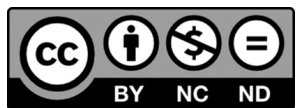

This work is licensed under a Creative Commons Attribution-NonCommercialNoDerivatives International License.

(C2021 The Editorial Committee of Annals of Thoracic and Cardiovascular Surgery
MN, USA) in the mitral position and a 23-mm Mosaic in the aortic position. With regard to the mitral valve, there was a large perforation and vegetation at the middle of the anterior leaflet, which was resected during surgery. Given the patient's presentation, we performed re-DVR because the valve in the mitral position showed structural valve deterioration leading to severe mitral regurgitation, as detected by echocardiography (Fig. 1a and 1b) and high pressure on Swan-Ganz catheterization. Both bioprostheses were extracted (Fig. 2) under cardiopulmonary bypass (CPB). In accord with the patient's choice, the replacement bioprosthetic valves were a 21-mm MagnaEASE (Edwards Lifesciences, Irvine, CA, USA) in the aortic position and a 29-mm Mosaic in the mitral position. At the mitral site, the old 27-mm Mosaic appeared to be implanted with everting sutures, concomitant with posterior leaflet preservation at the initial operation. This time, the new 29-mm Mosaic valve was inserted in supraannular position with non-everting sutures, concomitant with posterior leaflet resection. Weaning from CPB was smooth except for slightly high pressure in the pulmonary artery. While closing the chest, the hemodynamics remained stable, so the surgery was able to be completed uneventfully. As the patient was awakening from anesthesia in the intensive care unit (ICU), a large amount of bubble sputum appeared and continued to spout from the trachea tube. The blood pressure, which had gradually decreased, was restored by volume loading. Swan-Ganz 

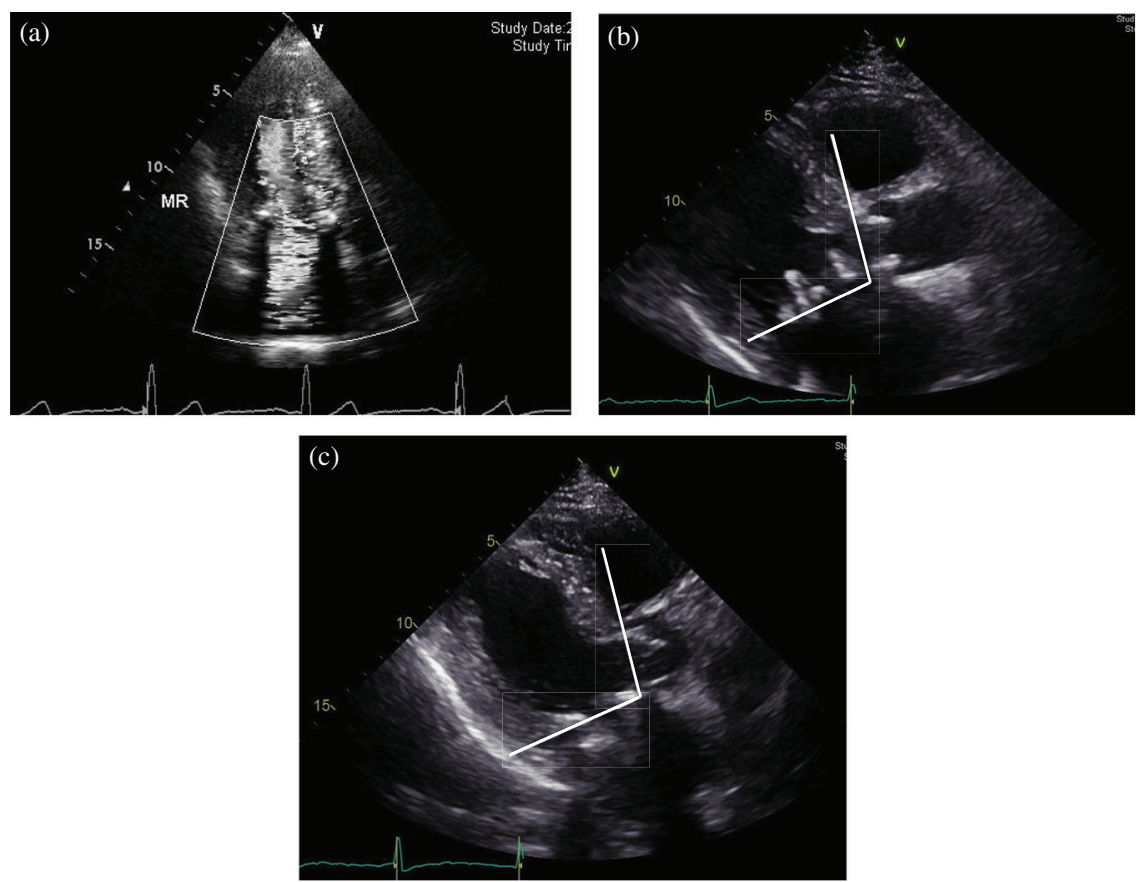

Fig. 1 (a) Preoperative echocardiography. Severe mitral regurgitation due to leaflet dehiscence. (b) Preoperative image. Narrowed aorto-mitral angle shown by two lines of each annular plane. (c) Postoperative echocardiography. The aorto-mitral angle is almost the same as the preoperative angle.

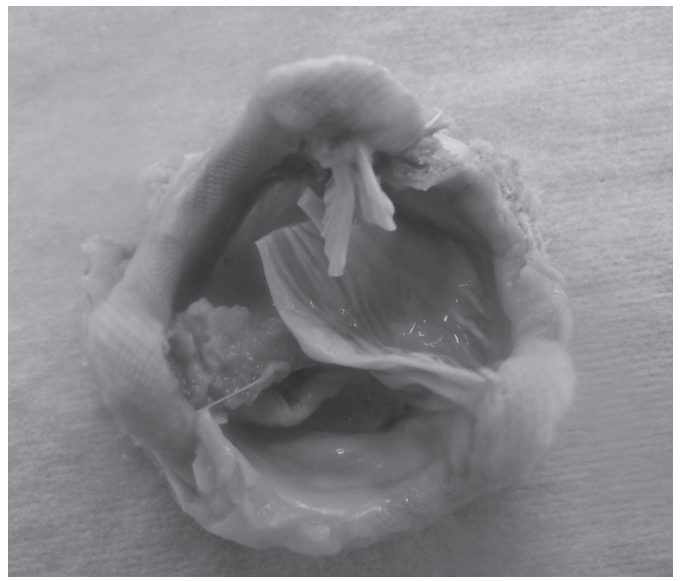

Fig. 2 The extracted Mosaic valve in the mitral position. The tear in the leaflet was detected.

data showed pulmonary artery pressure (PAP), central venous pressure $(\mathrm{CVP})$ and cardiac index $(\mathrm{CI})$ values of $47 / 22 \mathrm{mmHg}, 7 \mathrm{mmHg}$, and $2.1 \mathrm{~L} / \mathrm{min} / \mathrm{m}^{2}$, respectively, when the arterial pressure (AP) showed $70 / 29 \mathrm{mmHg}$, with the use of $0.02 \gamma$ of noradrenaline, $0.015 \gamma$ of epinephrine, $3 \gamma$ of dopamine, and $6 \gamma$ of dobutamine. The cardiologist performed transesophageal echocardiography (TEE). No MR was detected, and the status of LVOT could not be evaluated because of bioprosthesis artifact in the mitral position. Although we were unsure as to what had occurred, as there was a slight suspicion of LVOT obstruction caused by the bioprosthesis, we performed cardiac catheterization to achieve a definitive diagnosis and decide whether a re-operation was necessary. The pullout study revealed a significant pressure gradient (PG) between the left ventricle and the aorta. The left ventricular pressure was $200 \mathrm{mmHg}$, the LVOT pressure $160 \mathrm{mmHg}$, and the aortic pressure $100 \mathrm{mmHg}$ (Fig. 3), indicating that there was LVOT obstruction caused by the bioprosthesis. During the catheterization study, hemodynamic instability continued and intra-aortic balloon pumping (IABP) and percutaneous cardiopulmonary support (PCPS) were introduced. A second operation was immediately performed with re-sternotomy and conversion from PCPS to CPB. After extracting the implanted 29-mm Mosaic of the mitral position, a mechanical valve (SJM 27) was implanted. The postoperative course was satisfactory; PCPS was removed on postoperative day 1 (POD1) and IABP on POD3. The patient was extubated on POD6. Postoperative echocardiography showed no obstruction of the LVOT (Fig. 1c). The PG of trans-aorta showed $42 \mathrm{mmHg}$ (peak) and $21.2 \mathrm{mmHg}$ (mean), and the PG of $\mathrm{LV}$ outflow showed $5.0 \mathrm{mmHg}$ (peak) and $3.2 \mathrm{mmHg}$ (mean). The interventricular septum (IVS) measured 


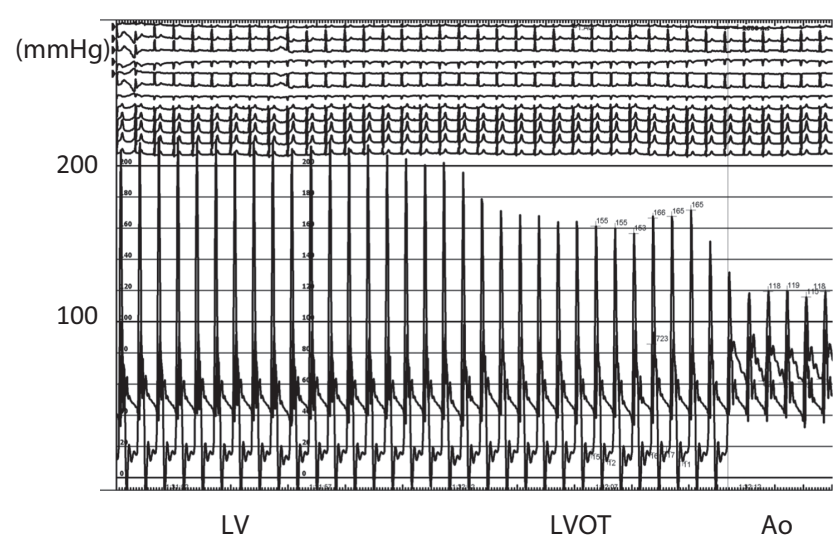

Fig. 3 Pullout pressure study from cardiac catheterization in the portion of the LV, the LVOT, and the Ao, showing a significant gradient among the Ao, the LVOT, and the LV. LV: left ventricle; LVOT: left ventricular outflow tract; Ao: aorta

$9.4 \mathrm{~mm}$ in diastolic phase and $12.0 \mathrm{~mm}$ in systolic phase. On the echo image, there was no muscular hypertrophy in the subaortic space, as is observed with hypertrophic cardiomyopathy.

\section{Discussion}

It has been well documented that the bioprosthetic valves can cause LVOT obstruction. ${ }^{1-3)}$ However, in recent years, the incidence of LVOT obstruction by bioprostheses has become rare, and there have been no reports of acute pulmonary edema caused by LVOT obstruction immediately after surgery. In this case, it was unexpected that the cause of pulmonary edema would be LVOT obstruction because weaning from the CPB during surgery was uneventful. Although we were always conscious of the possibility of LVOT obstruction related to the bioprosthesis, we needed to obtain definitive evidence before deciding to proceed to re-operation.

As a significant PG was recognized between the left ventricle and the aorta by cardiac catheterization, we decided to undertake prompt reoperation. The $100 \mathrm{mmHg}$ of PG between LV and Aorta was divided into $60 \mathrm{mmHg}$ and $40 \mathrm{mmHg}$ by pullout catheterization measurement through the subaortic space. We reasoned that the two stages of PG were the probable consequence of the subaortic space being surrounded by stent posts of the bioprosthesis of the mitral position. Diagnosis of LVOT obstruction by an artificial valve after cardiac catheterization has been rarely reported.4) During hemodynamic instability and the appearance of copious watery sputum, it ordinarily is difficult to transfer the patient from the
ICU to the catheter laboratory. However, a clear image on transthoracic echocardiography cannot be obtained after surgery because of the artifact in the LVOT created by the artificial valve in the mitral position. The TEE is more difficult to diagnose LVOT obstruction because it is difficult to detect good angle of flow Doppler at the LVOT. Consequently, a definitive diagnosis of LVOT obstruction is difficult to obtain via echo alone. One report in the literature proposes that the reduction or discontinuance of inotropic drugs and the use of $\beta$-blockers are useful for the treatment of LVOT obstruction. ${ }^{5)}$ In the present case, the patient's hemodynamics were collapsing, making it difficult to apply such drug management. Moreover, even if he could have remained stable without reoperation, the postoperative management would be fraught with difficulty.

The patient's status worsened after the surgery mainly because of awakening from anesthesia in the ICU. However, there had been some degree of influence by the LVOT obstruction after CPB weaning because the PAP was slightly higher than normal. The degree of LVOT obstruction might have been increased by intrinsic catecholamine.

In this case, the precise cause of the LVOT obstruction is debatable. One possibility is improper implantation. In this regard, the malrotation of the stent post on the bioprosthesis is one of the most critical points. Because this case involved re-implantation and native tissue of annulus was changed by the growth of fibrous tissue, the precise position of the trigon was not able to be identified. Therefore, we estimated the trigon's position based on the entire balance of the annulus. The stent post of the old prosthesis, which was extracted later, became the landmark, and the pledgets of the aortic annulus, which were visible through the mitral annulus, showed the direction of the LVOT. Therefore, we were able to evade the entire malrotation of $60^{\circ}$.

The other possibility is the high profile of the bioprosthesis. As the Mosaic valve has a high profile, theoretically the part of the stent post protruding toward the ventricular side is large. However, Jamieson et al. ${ }^{6}$ reported that there was no LVOT obstruction by Mosaic valve in the mitral position. It is also possible that the DVR was a contributory factor in causing LVOT obstruction. That is to say, in contrast to mitral valve replacement only, in DVR the aorto-mitral angle ${ }^{5)}$ might become narrower. We chose the same valve as used in the previous surgery for the mitral position because of its previous successful record. However, resection of the posterior 
leaflet and changing the suture procedure from everting mode to non-everting mode required a larger size of Mosaic valve. When the size of the valve was increased from 27 to $29 \mathrm{~mm}$, a larger protrusion forward of the outflow tract occurred. The height of the stent post of a $29 \mathrm{~mm}$ valve is $1.5 \mathrm{~mm}$ higher than that of a $27 \mathrm{~mm}$ valve. High protrusion of the stent post was apparent on the image of the preoperative echo study (Fig. 1b). Recently, a low-profile bioprosthesis has become available, introducing another option for future cases of valve replacement. We showed echocardiographically that the angle between the aortic annulus and mitral annulus, the so-called aorto-mitral angle, was almost the same preand postoperatively (Fig. 1b and 1c). If it were recognized preoperatively that the aorto-mitral angle was narrow, it would have been supposed that the stent post of the bioprosthesis widely covered the outflow tract and the LVOT obstruction would have been increased. In the case of DVR, bioprosthesis selection will be crucial because the aorto-mitral angle might be narrowed in comparison with single valve replacement.

\section{Conclusion}

We have presented a case of an acute pulmonary edema due to postoperative LVOT obstruction related to double valve re-replacement of bioprosthesis. DVR with bioprosthesis may cause the LVOT obstruction, and surgeons have to manage with paying attention for the LVOT. It is necessary to use of TEE and evaluate details of the LVOT carefully during valve replacement surgery.

\section{Disclosure Statement}

All authors have no conflict of interest about this study.

\section{References}

1) Esper E, Ferdinand FD, Aronson S, et al. Prosthetic mitral valve replacement: late complications after native valve preservation. Ann Thorac Surg 1997; 63: 541-3.

2) Tewari P, Basu R. Left ventricular outflow tract obstruction after mitral valve replacement. Anesthesia \& Analgesia 2008; 106: 65-66.

3) Bortolotti U, Milano A, Tursi V, et al. Fatal obstruction of the left ventricular outflow tract caused by lowprofile bioprostheses in the mitral valve position. Chest 1993; 103: 1288-9.

4) Aubert S, Neto OS, Pawale A, et al. Late mitral valve regurgitation after bullet wound to the heart. Ann Thorac Surg 2006; 82: 737-9.

5) Guler N, Ozkara C, Akyol A. Left ventricular outflow tract obstruction after bioprosthetic mitral valve replacement with posterior mitral leaflet preservation. Tex Heart Inst J 2006; 33: 399-401.

6) Jamieson WR, Riess FC, Raudkivi PJ, et al. Medtronic mosaic porcine bioprosthesis: assessment of 12-year performance. J Thorac Cardiovasc Surg 2011; 142: 302-7.e2. 